\title{
FULL PAPER \\ Surveillance of transcriptomes in basic military trainees with normal, febrile respiratory illness, and convalescent phenotypes
}

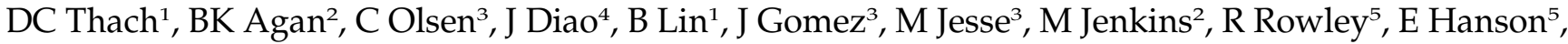 \\ C Tibbetts ${ }^{5,6}$, DA Stenger ${ }^{1}$, E Walter ${ }^{5,7}$ and EOS ${ }^{8}$ \\ ${ }^{1}$ Center for Biomolecular Science and Engineering, Naval Research Laboratory, Washington, DC, USA; ${ }^{2}$ Department of Infectious \\ Diseases, Wilford Hall USAF Medical Center, LAFB, TX, USA; ${ }^{3}$ Henry M Jackson Foundation, San Antonio, TX, USA; ${ }^{4}$ Spin Systems, \\ Inc., Sterling, VA, USA; ${ }^{5} \mathrm{HQ} U S A F / S G R$, Falls Church, VA, USA; ${ }^{6}$ The George Washington University, Washington, DC, USA; \\ ${ }^{7}$ Texas AEM University Systems, College Station, TX, USA
}

\begin{abstract}
Gene expression profiles permit analysis of host immune response at the transcriptome level. We used the Pax gene Blood RNA (PAX) System and Affymetrix microarrays (HG-U133A\&B) to survey profiles in basic military trainees and to classify them as healthy, febrile respiratory illness (FRI) without adenovirus, FRI with adenovirus, and convalescent from FRI with adenovirus. We assessed quality metrics of RNA processing for microarrays. Class prediction analysis discovered nested sets of transcripts that could categorize the phenotypes with optimized accuracy of $99 \%$ (nonfebrile vs febrile, $P<0.0005$ ), $87 \%$ (healthy vs convalescent, $P=0.001$ ), and $91 \%$ (febrile without vs with adenovirus, $P<0.0005$ ). The discovered set for classification of nonfebrile vs febrile patients consisted of 40 transcripts with functions related to interferon induced genes, complement cascades, and TNF and IL1 signaling. The set of seven transcripts for distinguishing healthy vs convalescent individuals included those associated with ribosomal structure, humoral immunity, and cell adhesion. The set of 10 transcripts for distinguishing FRI without vs with adenovirus had functions related to interferon induced genes, IL1 receptor accessory protein, and cell interactions. These results are the first in vivo demonstration of classification of infectious diseases via host signature transcripts and move us towards using the transcriptome in biosurveillance.
\end{abstract}

Genes and Immunity (2005) 6, 588-595. doi:10.1038/sj.gene.6364244; published online 21 July 2005

Keywords: transcriptome; surveillance; infection phenotypes

\section{Introduction}

Gene expression analysis using microarray technology holds the potential to advance our understanding of disease pathogenesis and to improve diagnosis, prognosis, and targeted therapeutics. Cancer research ${ }^{1,2}$ demonstrates that gene expression profiles can classify malignancies to a level of detail not previously possible, stratifying for prognosis and therapeutic response. Recently, profiles of blood cells from experimental smallpox infection of non-human primates were assessed to determine global immune responses to a pathogen. ${ }^{3}$ We further study the use of microarray technology to survey gene expression profiles from blood samples of a human population to determine whether specific immunologic signatures exist for a particular pathogen and to simultaneously elucidate pathways of pathogenesis at the transcriptome level.

Correspondence: $\operatorname{Dr} D$ Thach, Center for Biomolecular Science and Engineering, Naval Research Laboratory, 4555 Overlook Ave., SW Bldg 30, Washington, DC 20375, USA. E-mail: dthach@cbmse.nrl.navy.mil ${ }^{8}$ Epidemic Outbreak Surveillance (EOS) members listed at the end of paper.

Received 27 April 2005; revised 3 June 2005; accepted 6 June 2005; published online 21 July 2005
The setting for this study is Lackland Air Force Base (LAFB) in San Antonio, Texas, where Basic Military Trainees (BMTs) enter a 6-week training course and approximately $20 \%$ develop febrile respiratory illness (FRI). Since the discontinuation of the adenoviral vaccine in the late 1990s, approximately $60 \%$ of FRI is due to adenovirus serotype $4 .{ }^{4,5}$ We undertook a study to compare the gene expression profiles of healthy, FRI without adenovirus, FRI with adenovirus and those who had convalesced from FRI with adenovirus in this BMT population. Previously, we have shown methods for handling and processing blood specimens in which RNA integrity was maintained for transcriptome measurements. ${ }^{6}$ Now, we extend this experience to survey the gene expression profiles of this cohort to determine whether a set of transcripts exists that differentiate healthy from febrile and, specifically differentiate adenoviral from nonadenoviral causes of FRI.

\section{Results}

Clinical phenotypes

A total of 30 healthy, 19 with FRI and negative by culture for adenovirus, 30 with FRI and positive by culture for 
adenovirus, and 30 convalescing from adenoviruspositive FRI were enrolled in this study. Enrollees in these four infection status phenotypes were matched for age \pm 3 years and race/ethnicity. Only male BMTs were enrolled. After selection of samples meeting standards for gene expression analysis, 17 FRI without adenovirus had been ill for $5 \pm 3$ days (median \pm s.d.), whereas 26 FRI with adenovirus had been ill for $8 \pm 4$ days $(P=0.006$, Wilcoxon). The incidence of symptoms over all the groups was sore throat $(95.3 \%)$, cough $(93 \%)$, sinus congestion $(90.7 \%)$, headache $(88 \%)$, chills $(84 \%)$, rhinorrhea $(81 \%)$, body aches $(65 \%)$, malaise $(63 \%)$, nausea $(54 \%)$, diarrhea $(14 \%)$, pleuritic chest pain $(14 \%)$, vomiting $(14 \%)$, and rash $(0 \%)$, with no significant differences between the FRI groups. There was also no significant difference in allergies, recent injuries, and smoking history among the infection status phenotypes.

\section{Quality and variations of RNA derived from PAX system from the BMT population}

In order to identify clinically relevant gene expression profile differences for phenotypes in a population, it is essential that the RNA sample applied to the microarray is representative of the amount of transcripts in vivo. The PAX system was used to minimize handling of blood cells postcollection and to immediately stabilize RNA and halt transcription. We previously have shown two methods using this PAX system that provide stable RNA for microarray analysis. ${ }^{6}$

To assess RNA quality on each of the 95 microarrays analyzed in this study, recently published metrics derived from electropherograms of the RNA were used. ${ }^{7}$ Assessment of the degradation factor, which is the ratio of the average intensity of bands of lesser molecular weight than the $18 \mathrm{~S}$ ribosomal peak to the $18 \mathrm{~S}$ band intensity multiplied by 100, demonstrated minimal degradation of RNA (Figure 1). This degradation factor for the samples correlated with gapdh $3^{\prime} / 5^{\prime}$ on the A arrays (Figure 1c; $r=0.3, P=0.008$, ANOVA) and actin $3^{\prime} / 5^{\prime}$ on the B arrays $(r=0.2 ; P<0.05$, ANOVA), the internal measurements for assessment of RNA quality on the microarray. There was no significant correlation between 28S/18S vs degradation factor, gapdh $3^{\prime} / 5^{\prime}$, and actin $3^{\prime} / 5^{\prime}$, suggesting that the degradation factor is a superior method for assessing RNA quality for microarray analysis. No significant difference in degradation factor was seen among the phenotype groups.

Assessment of the apoptosis factor, which is the ratio of the height of the $28 \mathrm{~S}$ to $18 \mathrm{~S}$ peak, ${ }^{7}$ suggested that a high percentage of blood cells underwent apoptotic cell death. The distribution of the degradation factor, apoptosis factor, 28S/18S, and yields of total RNA are shown in Figure 1b. No significant difference in apoptosis factor was seen among the phenotype groups. There was no significant correlation between duration of freezing and degradation factor (Figure 1d), nor was there correlation with apoptosis factor, RNA yield, 28S/18S, or gapdh and actin $3^{\prime} / 5^{\prime}$.

We determined if blood cell type heterogeneity affected the sensitivity of transcript detection. Assessment of complete blood count (CBC) variables that affect the number of present calls on the microarray demonstrated a linear correlation between number of probesets called Present and Mean Corpuscular Hemoglobin $(\mathrm{MCH})$. A significant effect was detected $(r=0.272$;
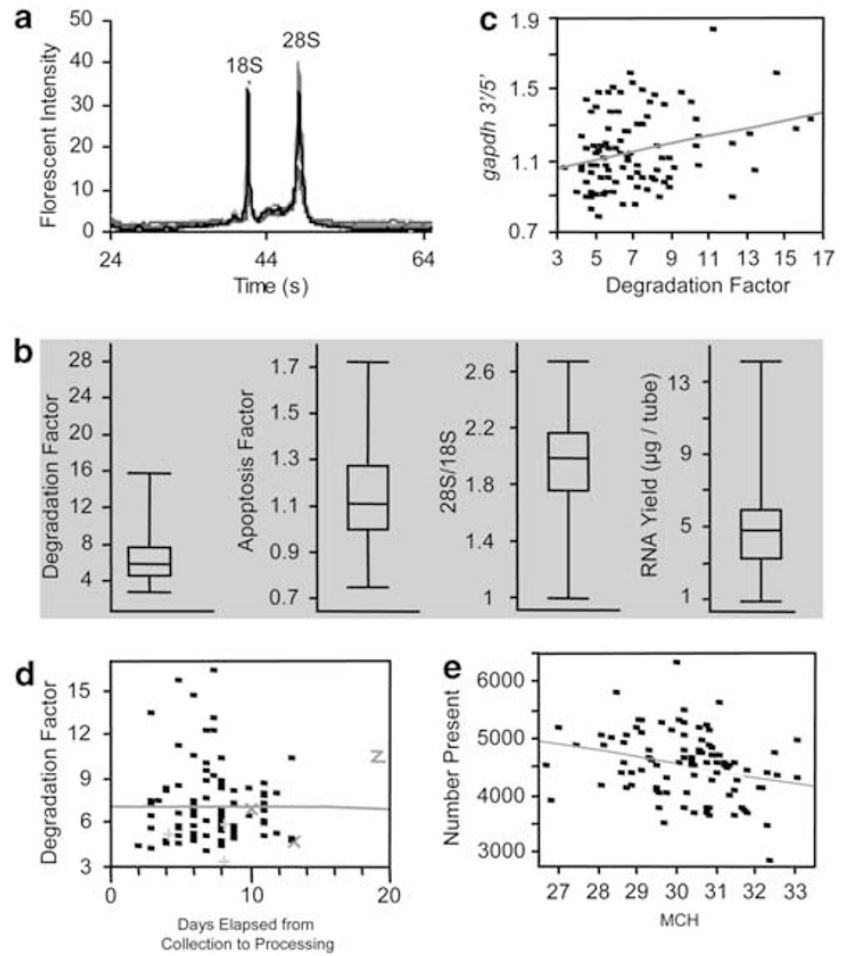

Figure 1 Quality of RNA derived from the PAX system of samples from the BMT population. (a) Overlay of electropherograms from BMTs with various phenotypes and handling conditions. The $18 \mathrm{~S}$ and $28 \mathrm{~S}$ ribosomal peaks are indicated. (b) Box plots of quality metrics calculated from the electropherograms. (c) Correlation between gapdh $3^{\prime} / 5^{\prime}$ values on the A arrays $v s$ degradation factor $(r=0.3, P=0.008$, ANOVA). (d) Lack of RNA degradation over days elapsed from blood collection to processing. Samples marked by ' + ', ' $x$ ', or ' $z$ ' had an additional freeze thaw cycle before final thawed for RNA isolation. (e) Correlation between the mean corpuscular hemoglobin $(\mathrm{MCH})$ and number of probesets called Present in the B arrays ( $r=-0.272 ; P=0.008$, ANOVA). Line shown is from equation: number Present $=8108-117 \mathrm{MCH}$.

$P=0.008$, ANOVA) for the B arrays only (Figure 1e). The equation of the regression line suggested that for every picogram increase in hemoglobin, there is a loss in present detection calls of 100 probesets or $2 \%$ of the average number of present called probesets on the B arrays. There was no difference in $\mathrm{MCH}$ among the infection status phenotypes.

\section{Quality of microarray measurements of PAX} system-derived RNA from the BMT population Individual control charts $v s$ the date of microarray scanning were plotted to establish stability of quality metrics over time, determine outliers, and compare with values proposed by the array manufacturer. The percentage of transcripts present was $32 \pm 10$ (average \pm 3 s.d.) for A arrays and $21 \pm 6$ for B arrays. The gapdh and actin $3^{\prime} / 5^{\prime}$ values were less than three, the upper-limit proposed by Affymetrix. ${ }^{8}$ Noise was $3.6 \pm 1.3$ for A arrays and $2.9 \pm 0.8$ for B arrays. Average Background was $100 \pm 48$ for A arrays and $78 \pm 33$ for B arrays. After exclusions of array sets that were known to have been processed differently or erroneously, a total of 95 A and B array sets with stable quality metrics remained. These 95 sets were processed in batches with nearly equal 
representation of the four infection status phenotypes. Therefore, comparisons among these four groups should detect biological differences as these groups have similar variations due to processing.

\section{Gene expression profiles}

The gene expression profiles were displayed on a heatmap with hierarchical clustering of transcripts to characterize and visualize patterns in the profiles of our cohort (Figure 2). Initial examination revealed a large number of transcripts with high expression levels (Figure 2, orange bar) and a smaller number of transcripts with low expression levels (Figure 2, purple bar) in the febrile group compared to the nonfebrile healthy and convalescent patients. There were also transcripts that showed differences between healthy and convalescent patients (Figure 2, gray bar), while

\begin{tabular}{|c|c|c|c|}
\hline $\begin{array}{l}\text { Healthy } \\
\text { (week 1) } \\
(n=27)\end{array}$ & $\begin{array}{l}\text { Febrile Ad- } \\
\text { (week 2-3) } \\
(n=17)\end{array}$ & $\begin{array}{l}\text { Febrile Ad+ } \\
\text { (week 2-3) } \\
(n=26)\end{array}$ & $\begin{array}{l}\text { Convalescent } \\
\text { (week 6) } \\
(n=25)\end{array}$ \\
\hline
\end{tabular}

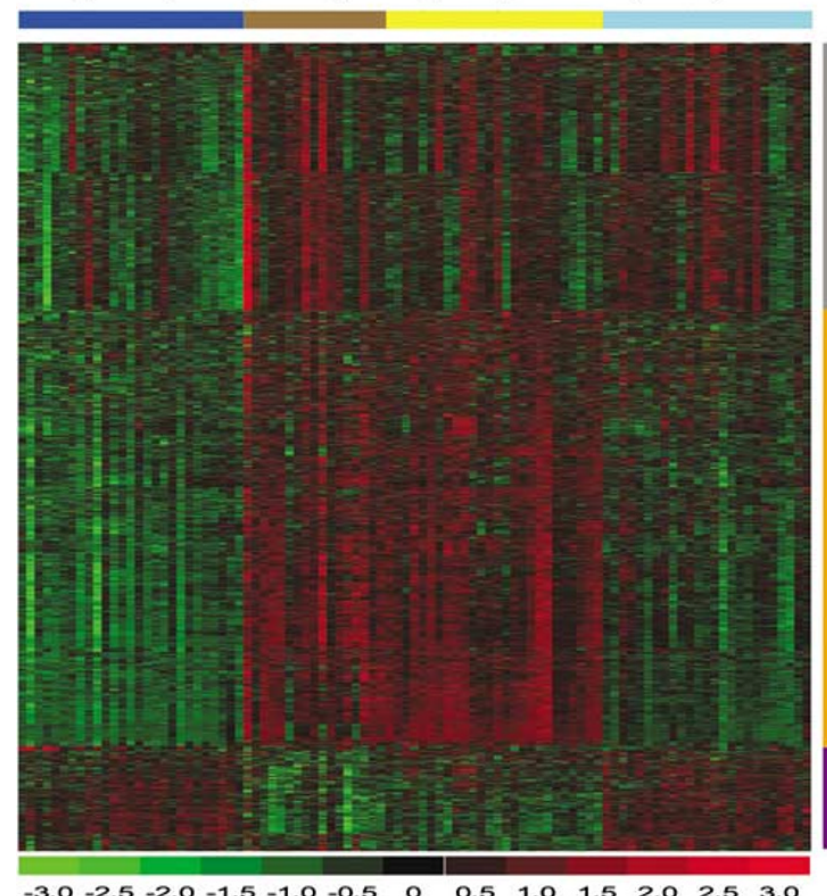

Figure 2 Gene expression profiles of the BMTs. To remove undetected transcripts, those with $>80 \%$ absent calls across samples were filtered resulting in 15721 from 44928 probesets. To remove uninformative transcripts, probesets in which less than $20 \%$ had a 1.5-fold or greater change from the probeset's median value were removed, resulting in 7682 probesets. To focus on transcripts with differences in expression among the four infection status phenotypes, those probesets with $P>0.01$ by ANOVA were excluded, resulting in 4414 probesets. The heat-map shows the transcript abundance (green to red intensities) detected by these 4414 probesets (rows) in each blood sample (column). The rows were hierarchically clustered with 1-correlation distance and average linkage, while the columns were sorted into the infection status phenotypes. Top blue, brown, yellow, and light blue bars denote samples from healthy, febrile without and with adenovirus, and convalescent patients, respectively. Bottom scale denotes standardized values for the green to red intensities in the heatmap. Side gray, orange, and purple bars denote clusters of transcripts that differ among the phenotypes. there was no obvious group of transcripts that showed differences between febrile without adenovirus $v$ f febrile with adenovirus from this visual inspection. Within each group, interindividual variation was observed, suggesting diverse immune responses in this population.

\section{Class prediction of infection status phenotype}

The pattern recognition above suggested that there were transcripts with differences in expression levels among healthy, febrile, and recovered patients. Therefore, class prediction was performed, to find sets of transcripts that best classify the four infection status phenotypes. Probesets with $>80 \%$ absent calls across samples were filtered resulting in 15721 probesets for further analysis. For supervised class prediction, the class labels for the febrile group were determine from respiratory viral culture results identifying presence or absence of adenovirus.

Figure 2 suggested that the fever status of individuals was the predominant source of variation in gene expression profiles among samples and this was confirmed by unsupervised clustering of samples. Thus, supervised class prediction analysis was used to find sets of transcripts that classified nonfebrile vs febrile patients first (node 1), then of the nonfebrile patients, further classified to healthy or convalescent (node 2), and among the febrile patients, further classified to without or with adenovirus infection (node 3 ). The segregation of the samples via this nodal scheme was confirmed via binary tree class prediction analysis.

Unlike data from cancer studies, ${ }^{2,9}$ there are no reported transcript selection methods or class prediction algorithms that are optimal for classification of infectious diseases. Therefore, we determined the transcript selection method and classification algorithm that would result in the highest percent correct classification during leave-one-out cross-validation. To estimate the optimal transcript selection parameters for classification in each node, the cutoff level of the univariate $P$-value was varied, selecting for probesets that showed statistically significant differences between the two groups at a $P$ value that was equaled to or smaller than a set cutoff level. As the $P$-value cutoffs became more stringent, the number of probesets selected decreased. For each $P$ value cutoff level, the selected probesets were subsequently used to classify the samples using various algorithms along with cross-validation analysis. For classification of node 1,2 and 3 , an optimal $P$-value cutoff level of $10^{-2}, 10^{-3}, 10^{-5}$ (Figure 3a-c, lower-left corner) was chosen, respectively.

Once an optimal $P$-value cutoff level was estimated and held constant, the additional criterion of fold-change cutoff threshold was varied (Figure $3 a-c, x$-axis) for each node. Figure 3 shows the percent-correct traces for the six algorithms tested tracking closely as fold-change cutoff level increases, but can differ by as much as $10-20 \%$ between methods. The black arrows in Figure 3 indicate an optimal percent-correct classification at the specific $P$ value and fold change cutoff. For nonfebrile $v$ s febrile, a percent correct call of $99 \%$ was achieved using the support vector machines algorithm at a $P$-value cutoff level of $10^{-2}$ and a fold-change threshold of $>5$ which selected for 47 probesets to be in the classifier (Figure 3a). For classification of healthy $v s$ convalescent patients, an optimal percent correct of $87 \%$ using the diagonal linear 

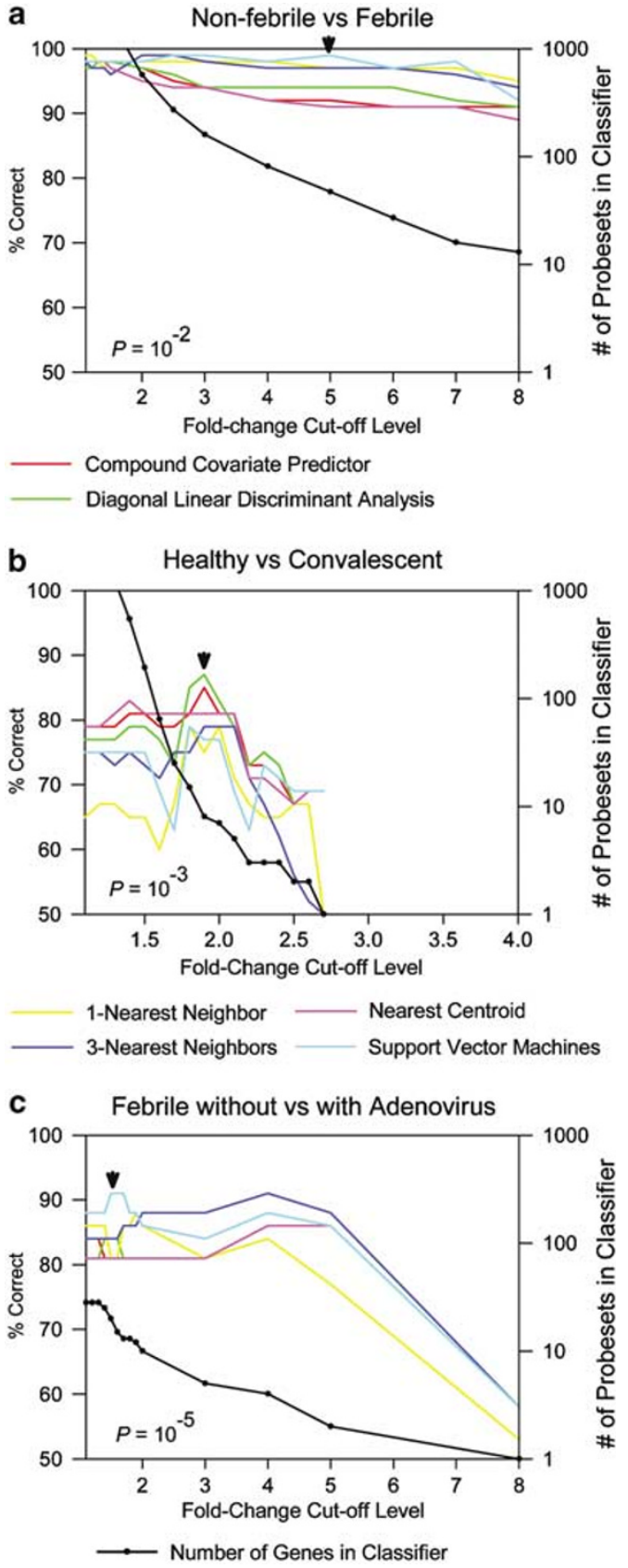

Figure 3 Optimization of class prediction for nonfebrile $v$ s febrile (a), healthy vs convalescent (b), and febrile without adenovirus vs febrile with adenovirus infection (c) phenotypes. Shown in the lower left corners of the three panels are the estimated optimal $P$ value cutoff levels for each of the three classifications. Classifier transcripts were further filtered by fold change level ( $x$-axis), with resulting percent correct classification (left $y$-axis) for various algorithms (color traces), and the number of probesets in the classifier (right $y$-axis, beaded black trace); arrows indicate fold change level that resulted in a highest percent correct classification.

discriminant analysis algorithm at a $P$-value cutoff level of $10^{-3}$ and a fold-change threshold of $>1.9$, which selected for eight probesets to be in the classifier was obtained (Figure 3b). For classification of febrile patients without- vs with adenovirus infection, an optimal percent-correct of $91 \%$ using the support vector machine
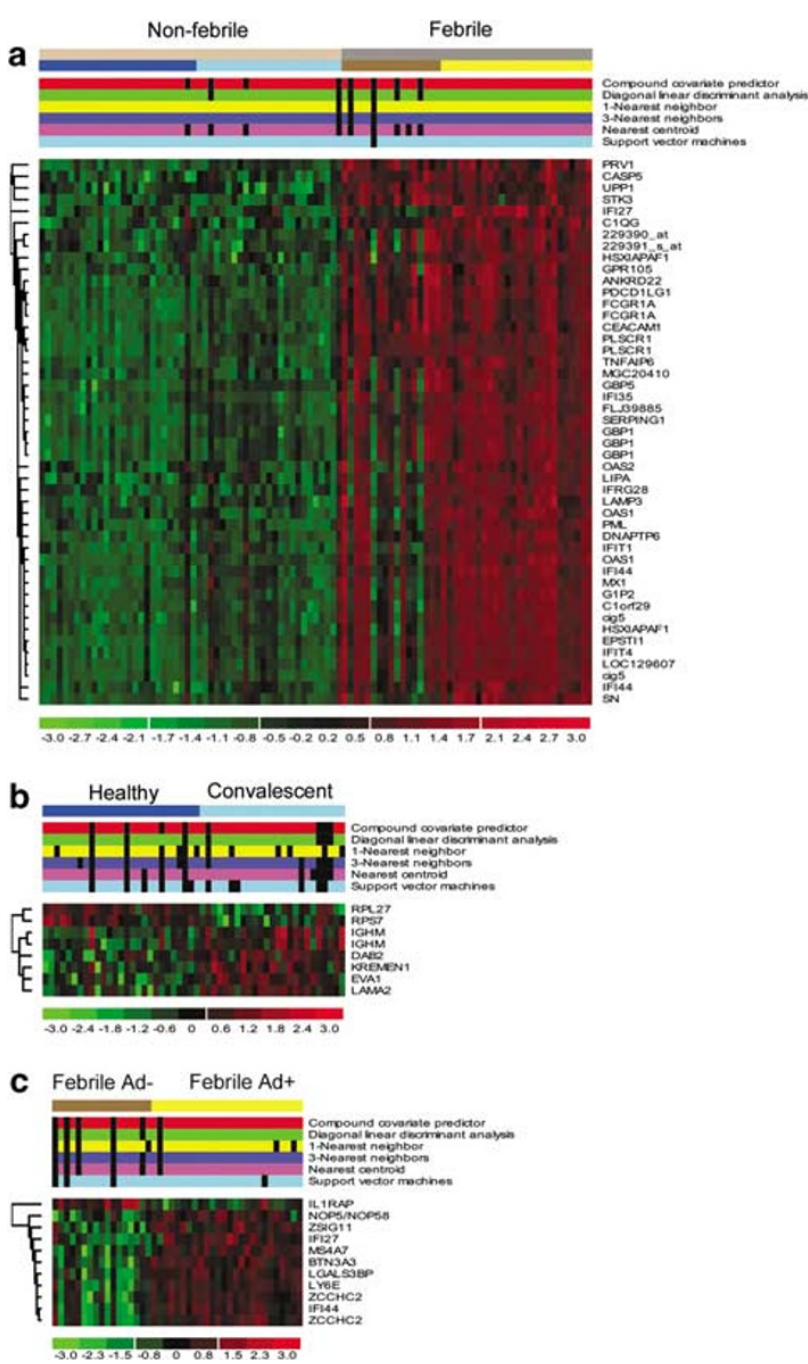

Figure 4 Identities and expression of genes in classifiers found from class prediction analysis. In each panel, top bar indicates the classification phenotypes of the samples (columns). Panel (a) has a second bar that further indicates healthy, convalescent, febrile without and with adenovirus samples as blue, light blue, brown, and yellow, respectively. The middle set of color bars in each panel mark samples that were misclassified (black) by various algorithms. The heat-maps indicate relative expression levels of genes (green to red intensities) identified by gene symbols on the right; for cDNA clones without gene symbols, probeset identifiers are displayed instead. Dendrograms are from clustering of standardized transcript levels (rows) using 1-correlation distance and average linkage. Bottom scale denotes standardized values for the green to red intensities in the heat-map. The transcript sets in panels (a)-(c) gave results marked by arrows in Figure $3 a-c$, respectively.

algorithm at a $P$-value cutoff level of $10^{-5}$ and a foldchange threshold of $>1.7$, which selected for 11 probesets to be in the classifier was obtained (Figure 3c).

The samples that were misclassified by various algorithms and the associated gene expression profiles for the selected transcript set are shown in Figure 4. For node 1 , no individuals were misclassified in the febrile with adenovirus group and misclassified samples tended to belong to the febrile without adenovirus or the convalescent group. For node 2, the misclassified samples seemed to be equally distributed between 
healthy and convalescent, while for node 3, the misclassified samples tended to be in the febrile without adenovirus group. One observes that some samples were misclassified regardless of algorithm.

The estimated optimal percent-correct classification of nonfebrile $v s$ febrile, healthy $v s$ convalescents, and febrile without vs with adenovirus infection patients were 99, 87 , and $91 \%$, respectively. To determine the reliability of these percentages, the permutation test was performed with 2000 permutations. This resulted in $P$-values of $<0.0005,0.001$, and $<0.0005$, respectively.

\section{Functions of genes in the classifier sets}

The identifiers of the discovered transcript sets for the class prediction results are shown in Figure 4. The 47 probesets used to classify fever status (Figure 4a) represent 40 transcripts. These included many that are induced by interferon, including: IFI27, IFI44, IFI35, IFRG28, IFIT1, IFIT4, OAS1, OAS2, GBP1, CASP5, MX1, and G1P2. Furthermore, OAS1 and OAS2 catalyze $2^{\prime}, 5^{\prime}$ oligomers of adenosine to activate RNaseL and inhibit cellular protein synthesis, while $M X 1$ is a member of the GTPase family. OAS1, OAS2, and MX1 have been shown to have antiviral functions, and interestingly, have also been found to be activated shortly after infection of nonhuman primates with high titers of smallpox. ${ }^{3}$ Transcripts involved in the complement cascade, C1QG which is downstream of antibody/antigen complexes and SERPING1 which inhibits activation of the first component of complement, were associated with fever. The TNF-alpha and IL-1 induced gene, TNFAIP6, which is a secretory protein involved in extracellular matrix stability and cell migration, and STK3 and CASP5, which are involved in the $M A P K$ signaling pathway and are downstream of the TNF and IL1 receptors were identified as class predictors. FCGR1A, which functions in the adaptive immune response and binds IgG, was part of the classifier. Other transcripts with associated known functions less clearly related to FRI or with unknown functions were also identified. Some gene ontology descriptions and, in parenthesis, their ratios of observed to expected number of occurrences were as follows: GTP binding (6), guanyl nucleotide binding (6), response to virus (32), immune response (8), defense response (7), response to pest/pathogen/parasite (6), and response to stress (3).

The eight probeset classifier for distinguishing healthy vs convalescent patients mapped to seven transcripts, including RPl27 and RPS7 associated with ribosomal structure; IGHM, the immunoglobulin heavy constant mu transcript; $L A M A 2$, which is involved with cell adhesion, migration, and tissue remodeling; and transcripts related to other functions such as DAB2, KREMEN1, and EVA1.

The 10 transcript classifier for distinguishing febrile without adenovirus vs with adenovirus infection included the interleukin-1 receptor accessory protein, IL1RAP; two interferon induced genes, IFI27 and IFI44, which were also in the classifier for fever status; and LGALS3BP, which is involved in cell-cell and cell-matrix interactions and has been found elevated in individuals infected with the human immunodeficiency virus. Other transcripts with known functions less clearly related to adenoviral FRI or with unknown functions included
ZCCHC2, ZSIG11, NOP5/NOP58, MS4A7, LY6E, and BTN3A3.

\section{Discussion}

After having rigorously assessed the RNA quality of samples processed with PAX tubes in a relatively large sample of humans with differing infection status phenotypes, we characterized and compared the transcriptomes from whole-blood samples of healthy, FRI without and with adenovirus infection, and convalescent individuals, evaluated class prediction methodologies, discovered nested sets of transcripts that could optimally classify the infection status phenotypes and have begun to implicate pathways and gene functions involved in FRI.

We applied a previously reported quality control metric called the degradation factor ${ }^{7}$ to our RNA samples and determined that this factor correlates with quality control metrics (gapdh $3^{\prime} / 5^{\prime}$ and actin $3^{\prime} / 5^{\prime}$ ) present on the microarray. This degradation factor can easily be applied to microarray studies on large populations by assessing electropherogram data that is available from a bioanalyzer prior to processing microarrays and an indicator can be set to flag poor quality samples. We find that quality metrics typically used, such as the 28S/ $18 \mathrm{~S}$ ratio have high variability outside the traditional standard range of 1.8-2.1 and poorly correlate with the quality control metrics present on the microarray.

When assessing signal-to-noise quality metrics, we discovered that $\mathrm{MCH}$ significantly affects number of present calls on the B array only, likely due to detection of low expression transcripts on the B array compared to the $\mathrm{A}$ array. ${ }^{10}$ At the time of probe design, the probes on the A chip were associated with more annotation than those on the $\mathrm{B}$ chip. The $\mathrm{MCH}$ is a measure of picograms of hemoglobin per red blood cell and likely is directly related to amounts of globin mRNA in whole-blood samples; prior studies have demonstrated that spiking of increasing amounts of globin mRNA transcripts into total RNA from a cell line decreases the percent present calls linearly. ${ }^{11}$ This factor would need to be controlled in future microarray studies or globin mRNA would need to be reduced. In the present study, there was no difference of $\mathrm{MCH}$ among the infection status phenotypes.

During supervised analysis, we varied the fold-change cutoff threshold in addition to the $P$-value cutoff to optimize percent correct classification. These combined criteria select for transcripts that not only are statistically different between two groups, but also vary above a specific fold-change threshold, reducing transcripts that may represent noise. The accuracy of classification seemed to be resistant to transcript selection parameters and algorithms when the gene-expression profiles showed large consistent differences, such as between nonfebrile $v$ s febrile patients; stricter $P$-value and fold change cutoff levels were needed to select informative transcripts that classify the healthy and convalescent or the febrile patients to an accuracy of 87 and $91 \%$, respectively.

Misclassified samples tended to belong to groups more likely to be heterogeneous, suggesting that the misclassification may be due to the lack of specificity of the class 
labels. In future studies of larger size, the convalescent group might be further subclassified based on duration of recovery and the febrile without adenovirus group subclassified based on specific pathogen identified. The majority of transcripts in the classifiers shown in Figure 4 remained in the classifier $100 \%$ of the time during leaveone-out cross-validation (100\% CV support). Thus, these transcripts in the classifiers are consistently different between individuals of two clinical phenotypes at the time when they present for study, as exemplified in Figure 4a. Individuals in the FRI with adenovirus group tend to present later in illness than those without potentially accounting for gene expression differences in the two groups. The correlation of changes in expression of these genes with infection status may also suggest that these genes are involved in the human host fever and immune responses to adenovirus infection in vivo. These transcripts consistently showed the largest fold changes between groups, suggesting that the changes in expression were at the pathway level and were unlikely to be accounted for by differences in cell concentration alone. Furthermore, there were no significant differences in cell-type concentration between the febrile without- vs with adenovirus groups. This correlation of transcripts to fever and immune responses was derived from in vivo natural infections of humans, suggesting the important role of these genes in the host response at the population level. Nested sets of transcripts resulted in similar percent-correct classifications, likely due to the fact that the expression of each transcript is not independent but correlated with other transcripts in related pathways. The discovery of transcripts with functions unrelated to immune response or with unknown functions implies that these should be further studied in infection phenotype model systems to elucidate mechanistic functions.

Our demonstration that one can predict the class of a patient with FRI due to adenovirus infection from background cases of FRI due to other etiologies support the possibility of using gene-expression in biosurveillance and pathogenesis. To our knowledge, this is the first in vivo demonstration of classification of infectious diseases via transcriptional signatures of the host. We intend to extend these findings to other respiratory pathogens, both viral and bacterial and to women, to further determine the capability of applying this technology to biodefense and infectious disease surveillance.

\section{Materials and methods}

\section{Entry criteria and sample collection}

LAFB is the location of Basic Military Training for all recruits to the United States Air Force. The BMTs are organized into flights of 50-60 individuals that eat, sleep, and train in close quarters. As many as 40-50 BMTs/ week present with FRI and 50-70\% are due to adenovirus. With approval of the LAFB IRB and after informed consent, approximately $15 \mathrm{ml}$ of blood, filling four to five PAX tubes, were drawn from each volunteer. On days 13 of training, blood was drawn from healthy BMTs into PAX tubes by standard protocol, ${ }^{12}$ but no nasal wash was collected for this group. During training, BMTs who presented with a temperature of $38.1^{\circ} \mathrm{C}$ or greater and FRI provided a nasal wash and blood draw. These individuals were categorized into either the FRI without adenovirus or with adenovirus group. Approximately 3 weeks after sample collection from the FRI volunteers with adenovirus, additional blood and nasal wash were collected to constitute samples for the convalescent group. All PAX tubes were maintained at room temperature for $2 \mathrm{~h}$, then frozen at $-20^{\circ} \mathrm{C}$ and shipped on dry-ice to the Navy Research Laboratory in Washington, DC for processing. Nasal washes were performed using a standard protocol with $5 \mathrm{ml}$ of normal saline lavage of the nasopharynx followed by collection of the eluent in a sterile container. Nasal wash eluent was stored at $4^{\circ} \mathrm{C}$ for 1-24 $\mathrm{h}$ before being aliquotted and sent for adenoviral culture. All BMTs underwent standardized questionnaires before each sample collection. Healthy individuals were screened again acute medical illness within 4 weeks of arriving at basic training. BMTs were screened for race/ethnicity, allergies, recent injuries, and smoking history to assess confounding variables for gene expression. The duration and type of respiratory symptoms to include sore throat, sinus congestion, cough, fever, chills, nausea, vomiting, diarrhea, fatigue, body aches, runny nose, headache, chest pain and rash were recorded. A physical examination was recorded.

\section{Sample processing}

Blood collection and RNA isolation was performed using the PAX System, which consists of an evacuated tube (PAX tube) for blood collection and a processing kit (PAX kit) for isolation of total RNA from whole blood. ${ }^{13,14}$ The isolated RNA was amplified, labeled, and interrogated on the HG-U133A and HG-U133B Genechip ${ }^{\circledR}$ microarrays (Affymetrix), noted here as A and B arrays, respectively.

\section{Total RNA isolation from blood}

Frozen PAX tubes were thawed at room temperature for $2 \mathrm{~h}$ followed by total RNA isolation as described in the PAX kit handbook, ${ }^{15}$ but modified to aid in tight pellet formation by increasing proteinase $\mathrm{K}$ from 40 to $80 \mu \mathrm{l}$ ( $>600 \mathrm{mAU} / \mathrm{ml}$ ) per sample, extending the $55^{\circ} \mathrm{C}$ incubation time from 10 to $30 \mathrm{~min}$, and the centrifugation time to $30 \mathrm{~min}$ or more. The optional on-column DNase digestion was not carried out. Purified total RNA was stored at $-80^{\circ} \mathrm{C}$.

\section{Target preparation}

For more complete removal of DNA from purified RNA, duplicate RNA samples were pooled, followed by insolution DNase treatment using the DNA-free ${ }^{\mathrm{TM}}$ kit (Ambion). However, to facilitate removal of the DNase inactivating beads, the completed reaction was spun through a spin column (Qiagen, Cat\#79523), rather than attempting to pipette off the supernatant without disturbing the bead pellet. Subsequently, $1 \mu \mathrm{l}$ from each sample was run on the bioanalyzer (Agilent) for assessment of RNA quality and quantity. The usage of the bioanalyzer was analogous to capillary gel electrophoresis. This resulted in electropherograms displaying florescent intensity $v$ s time (Figure 1a), which correlates with the amount of RNA vs the size of RNA, respectively. Next, $5 \mu \mathrm{g}$ of RNA were concentrated via ethanol precipitation as previously described. ${ }^{6}$ All subsequent steps were as described in the GeneChip Expression Analysis Technical Manual version 701021 Rev. 3. 


\section{Database integration}

The database consisted of clinical data such as information transcribed from standardized questionnaires, the $\mathrm{CBC}$, and the handling of blood samples. Laboratory data contained information about the processing of samples, from blood in PAX tubes to RNA extraction, as well as subsequent bioanalyzer measurements. Electropherograms were analyzed by the Biosizing (Agilent) software to output $28 \mathrm{~S} / 18 \mathrm{~S}$ intensity ratios and RNA yields, and by the Degradometer $1.1^{7}$ software to consolidate, scale, and calculate degradation and apoptosis factors. Report files summarizing the quality of target detection for an array were generated by GeneChip $^{\circledR}$ Operating Software 1.1 (Affymetrix). JMP (SAS) was used to join these various data tables together into a metadata table with more than a thousand columns. For gene-expression data, signal values were calculated using the Microarray Suite 5.0 algorithm with no scaling or normalization. This allows for subsequent testing of various scaling and normalization methods.

\section{Statistical analysis}

Statistical quality control and relations among metadata variables were analyzed in JMP. ANOVAs and class prediction of phenotypes using gene-expression data were performed in Arraytools 3.2.0 Beta developed by Richard Simon and Amy Lam (http:/ /linus.nci.nih.gov/ BRB-ArrayTools.html). Heat-maps and dendrograms were graphed using dChip. ${ }^{16,17}$ Analysis of gene functions was aided by Arraytools and EASE. ${ }^{18}$ Data analysis was performed primarily by DT.

Scaling was carried out for gene-expression data. For each blood sample, the same hybridization cocktail went onto the $\mathrm{A}$ and then the $\mathrm{B}$ array, allowing concatenation of the data from the two arrays to form a virtual array. This bypassed issues with analyzing the two data sets separately. The 100 control probesets common between the A and B arrays were selected based on stability in expression from a large study of various tissue types. ${ }^{10}$ Thus, all array data were scaled to a target value of 500 using the trimmed mean of the 100 control probesets. This resulted in stable Scale Factors (SF) over time and no differences in SF among the infection status phenotypes (ANOVA, $P=0.1047 \mathrm{~A}$ arrays, $P=0.1782 \mathrm{~B}$ arrays). This scaling method allowed for the concatenation of corresponding $\mathrm{A}$ and $\mathrm{B}$ arrays and should also remove variations that are not gene-specific.

\section{Acknowledgements}

We thank the study participants and F Ligler and J Golden for reviewing the manuscript. This work was supported in part by the Defense Threat Reduction Agency, HQ USAF Surgeon General's Office, Office of Naval Research, and the Naval Research Laboratory. The opinions and assertions contained herein are the private ones of the authors and are not to be construed as official or reflecting the views of the Department of Defense. The EOS Consortium is an Air Force Medical Service initiative comprised of: Sponsorship: P Demitry ${ }^{1}, \mathrm{~T}^{\mathrm{T}}$ Difato ${ }^{1}$; Executive Board: E Hanson ${ }^{4}$, R Holliday ${ }^{2}$, R Rowley ${ }^{4}, \mathrm{C}$ Tibbetts Operational Board: D Stenger ${ }^{10}$, E Walter $^{5}$, J Diao ${ }^{2}$; Technical Advisors $\mathcal{E}$ Collaborators: R Kruzelock ${ }^{6}$, B Agan ${ }^{10}$, L Daum $^{11}$, D Metzgar ${ }^{12}$, D Niemeyer ${ }^{11}$, K Russell ${ }^{12}$; Research $\mathcal{E}$
Clinical Staff: M Archer ${ }^{9}, \mathrm{R}_{\text {Bravo }^{3}}$, N Freed ${ }^{12}$, J Fuller ${ }^{12}$, J Gomez ${ }^{3}$, K Gratwick ${ }^{12}$, M Jenkins ${ }^{10}$, M Jesse ${ }^{3}$, B Johnson ${ }^{3}$, E Lawrence ${ }^{3}$, B Lin ${ }^{8}$, C Meador ${ }^{9}$, H Melgarejo ${ }^{3}$, K Mueller', C Olsen ${ }^{2}$, D Pearson ${ }^{3}$, A Purkayastha ${ }^{2}$, $\mathrm{J} \mathrm{Santiago}^{3}$, D Seto ${ }^{7}$, F Stotler ${ }^{3}$, D Thach $^{8}$, J Thornton ${ }^{9}$, Z Wang ${ }^{8}$, D Watson ${ }^{3}$, S Worthy ${ }^{3}$, G Vora ${ }^{8}$; Operations Support Staff: K Grant ${ }^{2}$, C James ${ }^{2}$. Affiliations: Dept. of ${ }^{1}$ USAF/SGR, ${ }^{2}$ USAF/SGR (Ctr), ${ }^{3}$ Lackland AFB, ${ }^{4}$ George Washington University, ${ }^{5}$ Texas A\&M University Systems, ${ }^{6}$ Virginia Tech, ${ }^{7}$ George Mason University, ${ }^{8} \mathrm{Naval}$ Research Laboratory, ${ }^{9}$ NOVA Research Incorporated, ${ }^{10}$ Wilford Hall Medical Ctr, ${ }^{11}$ Air Force Institute for Operational Health, ${ }^{12}$ Navy Health Research Ctr.

\section{References}

1 Bullinger L, Dohner K, Bair E et al. Use of gene-expression profiling to identify prognostic subclasses in adult acute myeloid leukemia. N Engl J Med 2004; 350: 1605-1616.

2 Valk PJ, Verhaak RG, Beijen MA et al. Prognostically useful gene-expression profiles in acute myeloid leukemia. $N$ Engl J Med 2004; 350: 1617-1628.

3 Rubins KH, Hensley LE, Jahrling PB et al. The host response to smallpox: analysis of the gene expression program in peripheral blood cells in a nonhuman primate model. Proc Natl Acad Sci USA 2004; 101: 15190-15195.

4 Barraza EM, Ludwig SL, Gaydos JC, Brundage JF. Reemergence of adenovirus type 4 acute respiratory disease in military trainees: report of an outbreak during a lapse in vaccination. J Infect Dis 1999; 179: 1531-1533.

5 Gray GC, Goswami PR, Malasig MD et al. Adult adenovirus infections: loss of orphaned vaccines precipitates military respiratory disease epidemics. For the Adenovirus Surveillance Group. Clin Infect Dis 2000; 31: 663-670.

6 Thach DC, Lin B, Walter E et al. Assessment of two methods for handling blood in collection tubes with RNA stabilizing agent for surveillance of gene expression profiles with high density microarrays. J Immunol Methods 2003; 283: 269-279.

7 Auer H, Lyianarachchi S, Newsom D et al. Chipping away at the chip bias: RNA degradation in microarray analysis. Nat Genet 2003; 35: 292-293.

8 Affymetrix. GeneChip ${ }^{\mathbb{B}}$ expression analysis data analysis fundamentals. Part No. 701190 Rev. 4. p 39. Available at http://www. affymetrix.com/support/downloads/manuals/data_analysis fundamentals_manual.pdf. Accessed Sept. 2004, 2004.

9 Golub TR. Toward a functional taxonomy of cancer. Cancer Cell 2004; 6: 107-108.

10 Affymetrix. Performance and validation of the GeneChip ${ }^{R}$ human genome U133 set. Available at http://www.affymetrix.com/support/technical/technotes/hgu133_performance_ technote.pdf. Accessed March 2005, 2002.

11 Affymetrix. Globin reduction protocol: a method for processing whole blood rna samples for improved array results. Available at http://www.affymetrix.com/support/technical/ technotes/blood2 technote.pdf. Accessed March 2005, 2003.

12 Preanalytix. Product circular. PAXgene Blood RNA tube Available at http://www.preanalytix.com/pdf/prodcir.pdf. Accessed Sept. 2004.

13 Jurgensen S, Schram J, Herdman C, Rainen L, Wyrich R, Oelmueller U. Effect of blood collection and storage conditions on gene expression analysis. Available at http://www. preanalytix.com/pdf/SJAMPposterNov01.pdf. Accessed April 2003.

14 Jurgensen S, Schram J, Herdman C, Rainen L, Wyrich R, Oelmueller U. New technology to stabilize cellular RNA in blood. Available at http://www.preanalytix.com/pdf/ AMP2000PosterSJ.pdf. Accessed April 2003. 
15 Preanalytix. PAXgene blood RNA kit handbook. Available at http://www.preanalytix.com/pdf/RNA_handbook.pdf. Accessed Sept 2004.

16 Li C, Hung Wong W. Model-based analysis of oligonucleotide arrays: model validation, design issues and standard error application. Genome Biol 2001; 2 RESEARCH0032.
17 Li C, Wong WH. Model-based analysis of oligonucleotide arrays: expression index computation and outlier detection. Proc Natl Acad Sci USA 2001; 98: 31-36.

18 Hosack DA, Dennis Jr G, Sherman BT, Lane HC, Lempicki RA. Identifying biological themes within lists of genes with EASE. Genome Biol 2003; 4: R70. 